\title{
Efectividad del programa "Healthy Kids" en los conocimientos, aptitudes y prácticas sobre alimentación saludable y actividad física para la prevención del sobrepeso y la obesidad en los estudiantes del $3^{\circ}$ grado de primaria del colegio Almirante Guillermo Brown, en el distrito de Villa El Salvador, 2011
}

\author{
Katering Leidy ALTAMIZA HUAMÁN y Neda POMÉ CÁRDENAS²
}

\begin{abstract}
RESUMEN
Objetivo: El objetivo de esta investigación es determinar la efectividad del programa"Healthy kids" en los conocimientos, aptitudes y prácticas sobre alimentación saludable y actividad física para la prevención de sobrepeso y obesidad en escolares de educación primaria. Metodología: El diseño es descriptivo con pre y post prueba. Se trabajó con escolares obesos y con sobrepeso que aprobaron los criterios de inclusión del estudio. La duración de la intervención fue de un mes e incluyó dos sesiones educativas para los niños con sus respectivos materiales didácticos de reforzamiento en casa y a las madres se les capacitó en dos sesiones. Resultados: Como resultado se obtuvo que el $52.9 \%$ de los escolares tiene diagnóstico nutricional normal, entretanto que, en sobrepeso y obesidad no están muy lejos $17,6 \%$ y $23.5 \%$ respectivamente, alcanzando así un $41.1 \%$ del total de escolares cuya cifra es de riesgo para la población estudiantil. Conclusiones: Se obtuvo un efecto positivo (0.001) en los conocimientos, actitudes y práctica de los escolares con respecto a alimentación saludable y actividad física.
\end{abstract}

Palabras clave: Alimentación, sobrepeso, obesidad, programa educativo.

\begin{abstract}
Objective: The aim of this investigation is to determine the efficiency of Program "Healthy kids" in healthy supply and physical activity for the prevention of overweight and obesity in students of primary education. Methodology: The design is descriptive with pre and post tries. One worked with obese students and with overweight that there approved the criteria of incorporation of the study. The duration of the intervention was 1 month and I include 2 educational meetings for the children with his respective didactic materials of reinforcement in house and I qualify the mothers in two meetings. Results: Since result obtained that $52.9 \%$ of the students has nutritional normal Diagnosis, meanwhile that overweight and obesity are not very far $17,6 \%$ and $23.5 \%$ respectively, reaching this way $41.1 \%$ of the total of students whose number is of risk for the student population. Conclusion: A positive effect (0.001) was obtained in the knowledge, attitudes and practice of the students with regard to healthy supply and physical activity.
\end{abstract}

Keywords: Feed, Overweight, obesity, educational program.

${ }^{1}$ Estudiante del $5^{\circ}$ año de Nutrición Humana, Universidad Peruana Unión.

${ }^{2}$ Magíster en Salud Pública, Docente de la Facultad de Ciencias de la Salud, Universidad Peruana Unión. 


\section{INTRODUCCIÓN}

Actualmente la obesidad es uno de los problemas de salud pública más relevantes del siglo XXI, según la Organización Mundial de la Salud (2006), indica que viene afectando de forma alarmante a la población infantil y adolescencia, sobre todo en países bajos y de medianos ingresos.

La obesidad es una enfermedad crónica y a la vez un factor de riesgo para desarrollar enfermedades metabólicas y cardiovasculares (Adolfo, Jáuregui \& Ruiz, 2007). Esta enfermedad se presenta tanto en adultos como en jóvenes y niños en edad escolar. La obesidad en la niñez es un fenómeno alarmante pues se conoce que del 25 al $50 \%$ de niños y adolescentes obesos, tienden a convertirse en adultos obesos. La obesidad es de origen multi causal; se identifican causas de origen genético, metabólico, conductual y ambiental. El consumo de alimentos ricos en grasa y la escasa actividad física son conductas que caracterizan los estilos de vida impuestos por las ciudades que ha afectado las costumbres y la salud de las familias. (Llanos \& Cabello, 2003).

La Organización Mundial de la Salud (2005), señala la necesidad de prevenir la obesidad desde las edades tempranas de la vida y promueve, entre otros aspectos, impulsar el consumo de dietas balanceadas e incrementar la actividad física, como medidas de autocuidado que promuevan la salud. En el cumplimiento de estos objetivos nosotros jugamos un papel sustantivo al responsabilizarnos de intervenciones que faciliten y promuevan una conducta de autocuidado que contribuya a mantener el mejor estado de salud factible en seres humanos.

La etapa escolar se considera oportuna para adoptar conductas de autocuidado a la salud, practicando un régimen alimentario saludable y ejercicio físico. En función a ello, el propósito del presente estudio fue determinar la efectividad de Healthy kids en alimentación saludable y actividad física para la prevención del sobrepeso y obesidad en los estudiantes del $3^{\circ}$ grado de primaria del colegio Almirante Guillermo Brown, en el distrito de Villa El Salvador.

\section{MATERIAL Y MÉTODOS}

Estudio cuasi experimental, descriptivo. Se usaron técnicas de muestreo no probabilístico, seleccionando a los estudiantes que cumplan los criterios de selección especificados como: pertenecer al tercer grado de primaria, escolares de edades comprendidas entre 7- 10 años de edad.

La muestra estuvo compuesta por 17 estudiantes escolares que proceden del Colegio Educativo Particular Almirante Guillermo Brown, $3^{\circ}$ grado de primaria Villa El Salvador.

El peso y la talla fueron datos que se obtuvieron con un tallímetro (SENAN) y balanza digital calibrada. El diagnóstico nutricional para los adultos se obtuvo a través de la las tablas de valoración nutricional según IMC (20 a 59 años de edad). (OMS, 2000).

Para obtener el diagnóstico nutricional de los escolares se utilizaron las tablas de valoración nutricional según IMC (5 a 19 años) para respectivos sexos. (Desviación Estándar, OMS.).

El estudio estadístico se realizó con el paquete SPSS 15.0, aplicaron estadísticos simples; tamaño de la muestra y porcentaje para las cualitativas. La comparación entre grupos se realizó mediante la prueba $\mathrm{T}$ para muestras relacionadas (sigma menor 0.05 ). Se obtuvo información a través de encuestas elaboradas por la investigadora y sometidas a juicios de expertos. Las esncuestas se tomaron a escolares y madres de familia previamente validadas, con dimensiones de alimentación, actividad física midiendo los conocimientos, actitudes y prácticas de los participantes del programa educativo antes y después de las sesiones educativas. El programa educativo cursó por consecutivas fases, desde el contacto con la directora del plantel hasta la toma de la segunda encuesta, dentro de los cuales se iba desarrollando en el aula educativa a un determinado horario, los diferentes temas de capacitación a padres y niños con los respectivos materiales validados. 


\section{RESULTADOS}

\section{Figura 1}

\section{Diagnóstico nutricional según IMC de los escolares}

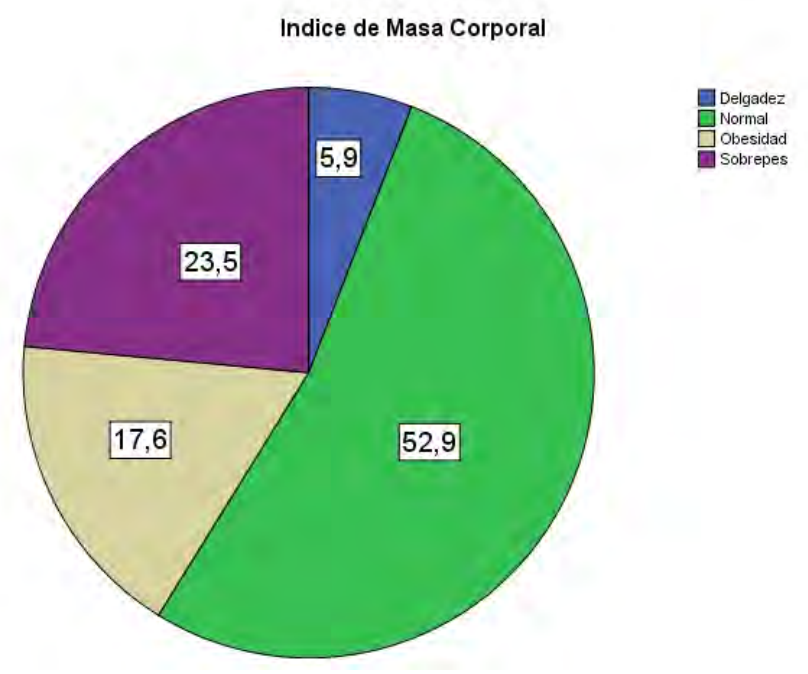

En la figura 1 se observa que el $52.9 \%$ de los escolares tiene diagnóstico nutricional normal, entretanto que en sobrepeso y obesidad no están muy lejos $17,6 \%$ y $23.5 \%$ respectivamente, alcanzando así un $41.1 \%$ del total de escolares cuya cifra es de riesgo para la población estudiantil, debido a que la alta prevalencia de esta malnutrición amenaza la salud de los escolares y su óptimo desarrollo.

\section{Tabla 1}

Diagnóstico nutricional (IMC según edad) de escolares

\begin{tabular}{|c|c|c|c|c|c|c|c|c|c|}
\hline \multicolumn{10}{|c|}{ TALLA/EDAD } \\
\hline & & \multicolumn{2}{|l|}{ Normal } & \multicolumn{2}{|c|}{ Riesgo de talla baja } & \multicolumn{2}{|c|}{ Talla baja } & \multicolumn{2}{|c|}{ Total } \\
\hline & & $\mathrm{n}$ & $\mathrm{N} \%$ & $\mathrm{n}$ & $\mathrm{N} \%$ & $\mathrm{n}$ & $\mathrm{N} \%$ & $\mathrm{n}$ & $\mathrm{N} \%$ \\
\hline \multirow[t]{5}{*}{ IMC } & Delgadez & 0 & $.0 \%$ & 0 & $.0 \%$ & 1 & $5.9 \%$ & 1 & $5.9 \%$ \\
\hline & Normal & 8 & $47.1 \%$ & 0 & $.0 \%$ & 1 & $5.9 \%$ & 9 & $52.9 \%$ \\
\hline & Obesidad & 2 & $11.8 \%$ & 1 & $5.9 \%$ & 0 & $.0 \%$ & 3 & $17.6 \%$ \\
\hline & Sobrepeso & 4 & $23.5 \%$ & 0 & $.0 \%$ & 0 & $.0 \%$ & 4 & $23.5 \%$ \\
\hline & Total & 14 & $82.4 \%$ & 1 & $5.9 \%$ & 2 & $11.8 \%$ & 17 & $100.0 \%$ \\
\hline
\end{tabular}

En la tabla 1 se observa que el 47.1\% con Índice de Masa Corporal normal se encuentra con talla adecuada para la edad. De forma similar el $23.5 \%$ de diagnóstico de sobrepeso. En tanto que $11.8 \%$ de escolares del $3^{\circ}$ año se encuentra con obesidad y con talla adecuada para la edad, no obstante un $5.9 \%$ se encuentra con riesgo de talla baja, lo cual confirman los estudios, en donde existe una relación de sobrepeso, obesidad y talla baja debido a múltiples factores, dentro de los que se encuentra la nutrición como factor determinante. 
Figura 2

Nivel de conocimientos de los alumnos del $3^{\circ}$ grado sobre alimentación saludable y actividad física antes y después de la intervención.

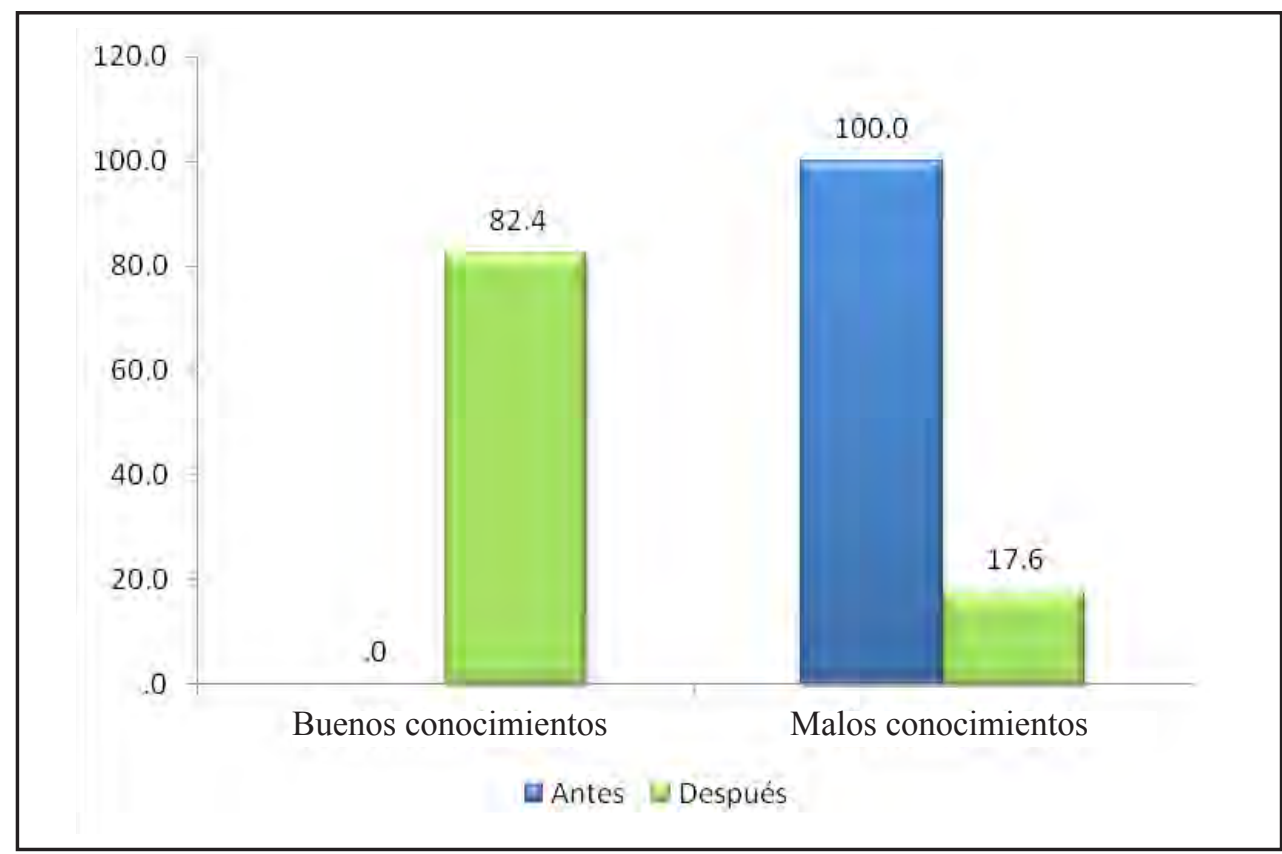

En la figura 2 se aprecia que antes del programa el 100\% de los escolares tenía malos conocimientos sobre alimentación saludable y actividad física y después de la intervención un 82.4\% mejoraron sus conocimientos.

Similares estudios, realizados en 10 escuelas públicas, mostraron que mejorando los conocimientos en los escolares en un período de dos años, se pudo obtener aumento en sus conocimientos, además de una significativa disminución del porcentaje de escolares con sobrepeso y obesidad.

Tabla 2

Análisi de prueba t para el nivel de conocimientos antes y después de la intervención del programa.

\begin{tabular}{|c|c|c|c|c|c|c|c|c|}
\hline \multicolumn{8}{|c|}{ Diferencias relacionadas } & $\begin{array}{c}\text { Sig. } \\
\text { (bilateral) }\end{array}$ \\
\hline \multirow{2}{*}{ Nivel } & \multirow[t]{2}{*}{ Media } & \multirow[t]{2}{*}{$\begin{array}{l}\text { Desviación } \\
\text { típica }\end{array}$} & \multirow[t]{2}{*}{$\begin{array}{l}\text { Error típica } \\
\text { Media }\end{array}$} & \multicolumn{2}{|c|}{$\begin{array}{l}95 \% \text { Intervalo de } \\
\text { confianza }\end{array}$} & \multirow[t]{2}{*}{$\mathrm{t}$} & \multirow[t]{2}{*}{$\mathrm{gl}$} & \\
\hline & & & & Inferior & Superior & & & \\
\hline $\begin{array}{l}\text { Puntaje de } \\
\text { conocimientos } \\
\text { obtenidos antes } \\
\text { y después del } \\
\text { programa }\end{array}$ & .82353 & .39295 & .09531 & .62149 & 1.02557 & 8.641 & 16 & .000 \\
\hline
\end{tabular}

En la tabla 2 el valor sigma de 0.000 de la prueba T para muestras relacionadas, muestra un valor muy significativo, el cual afirma que existe efectividad en la mejora de los conocimientos sobre alimentación saludable y actividad física en los escolares. 
Figura 3

Actitudes de los alumnos del $3^{\circ}$ grado sobre alimentación saludable y actividad física antes y después de la intervención

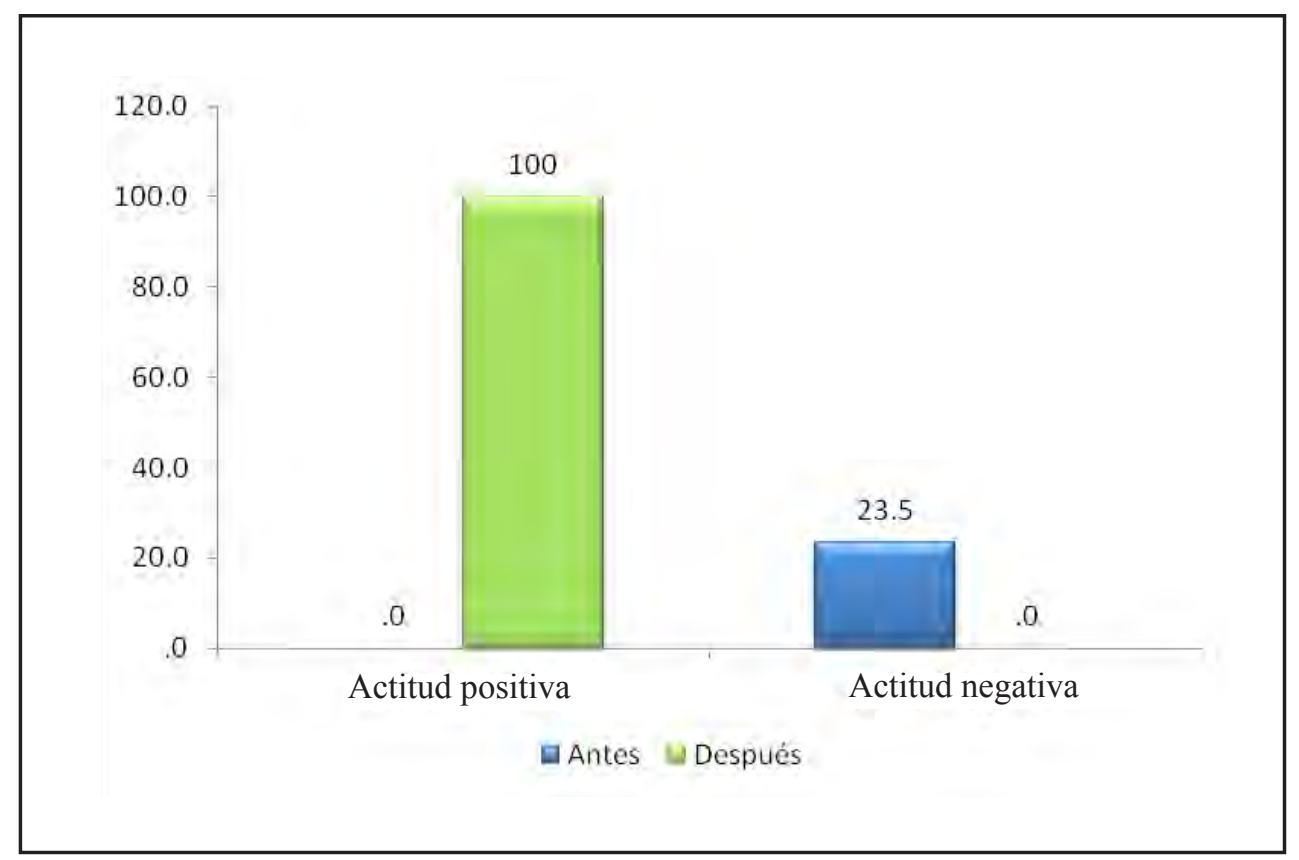

Como se observa en la figura 3, el 23.5\% de los escolares presentó actitudes negativas hacia la alimentación saludable y actividad física antes de la intervención, sin embargo después del programa el 100\% presentó una actitud positiva.

Tabla 3

Análisis de prueba t para las actitudes antes y después de la intervención del programa

\begin{tabular}{|c|c|c|c|c|c|c|c|c|}
\hline \multicolumn{8}{|c|}{ Diferencias relacionadas } & \multirow[t]{3}{*}{$\begin{array}{l}\text { Sig. } \\
\text { (bilateral) }\end{array}$} \\
\hline \multirow{2}{*}{ Nivel } & \multirow[t]{2}{*}{ Media } & \multirow[t]{2}{*}{$\begin{array}{c}\text { Desviación } \\
\text { típica }\end{array}$} & \multirow[t]{2}{*}{$\begin{array}{c}\text { Error típica } \\
\text { Media }\end{array}$} & \multicolumn{2}{|c|}{$\begin{array}{c}\text { 95\% Intervalo de con- } \\
\text { fianza }\end{array}$} & \multirow[t]{2}{*}{$\mathrm{t}$} & \multirow[t]{2}{*}{$\mathrm{gl}$} & \\
\hline & & & & Inferior & Superior & & & \\
\hline $\begin{array}{l}\text { Puntaje de } \\
\text { conocimientos } \\
\text { obtenidos antes } \\
\text { y después del } \\
\text { programa }\end{array}$ & .23529 & .43724 & .10605 & .01049 & .46010 & 2.219 & 16 & .041 \\
\hline
\end{tabular}

En esta tabla 3 el valor sigma de 0.041 de la prueba T para muestras relacionadas, obtuvo un valor significativo, el cual afirma que existió efectividad en las actitudes sobre alimentación saludable y actividad física. 


\section{Figura 4}

Nivel de prácticas de los alumnos del $3^{\circ}$ grado sobre alimentación saludable y actividad física antes y después de la intervención

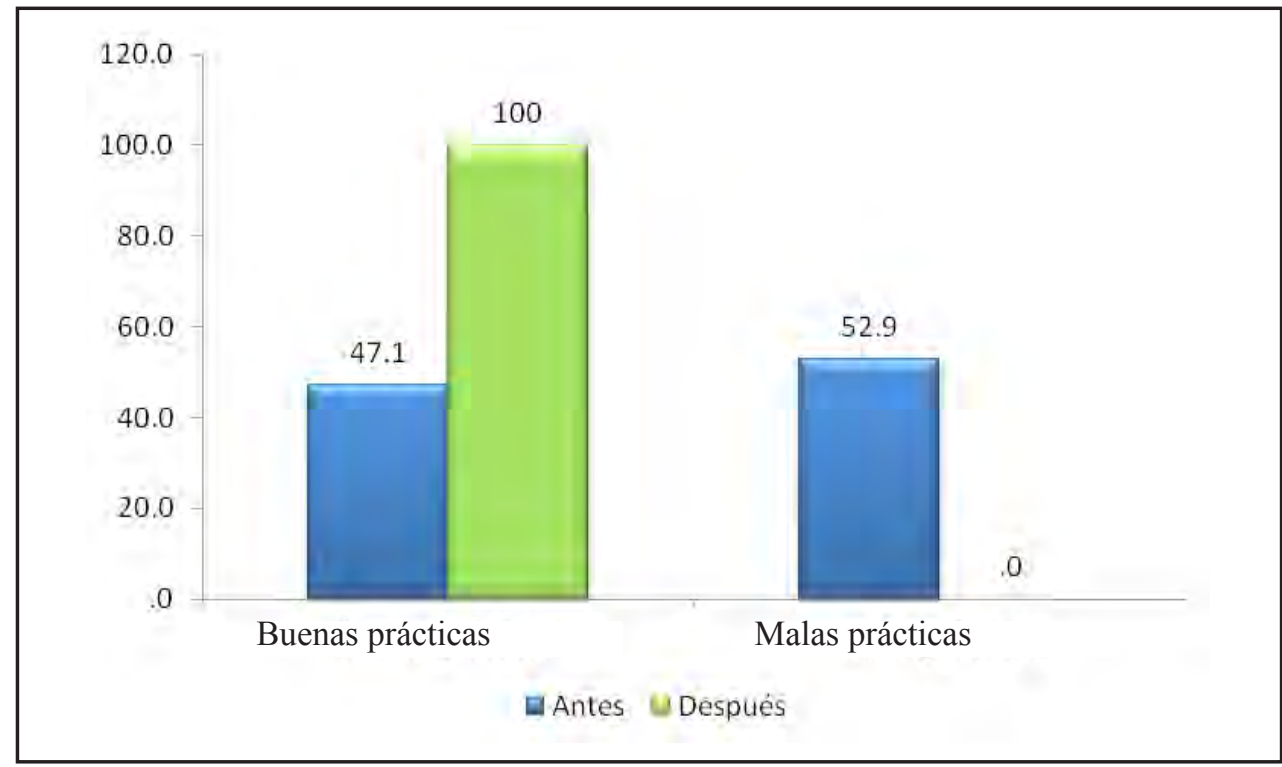

Como se observa en la figura 4, el 52.9\% de los escolares presentaron malas prácticas de alimentación saludable y actividad física antes de la intervención. Después del programa el 100\% tuvo prácticas buenas.

Tabla 4

Análisis de prueba t para las prácticas antes y después de la intervención del programa

\begin{tabular}{|c|c|c|c|c|c|c|c|c|}
\hline \multicolumn{8}{|c|}{ Diferencias relacionadas } & \multirow[t]{3}{*}{$\begin{array}{l}\text { Sig. } \\
\text { (bilateral }\end{array}$} \\
\hline \multirow{2}{*}{ Nivel } & \multirow[t]{2}{*}{ Media } & \multirow[t]{2}{*}{$\begin{array}{l}\text { Desviación } \\
\text { típica }\end{array}$} & \multirow[t]{2}{*}{$\begin{array}{l}\text { Error típica } \\
\text { Media } \\
\end{array}$} & \multicolumn{2}{|c|}{$\begin{array}{l}\text { 95\% Intervalo de con- } \\
\text { fianza }\end{array}$} & \multirow[t]{2}{*}{$\mathrm{t}$} & \multirow[t]{2}{*}{ gl } & \\
\hline & & & & Inferior & Superior & & & \\
\hline $\begin{array}{l}\text { Puntaje de } \\
\text { conocimientos } \\
\text { obtenidos antes } \\
\text { y después del } \\
\text { programa }\end{array}$ & .52941 & .51450 & .12478 & .26488 & .79394 & 4.243 & 16 & .001 \\
\hline
\end{tabular}

Realizando el análisis de la prueba t para muestras relacionadas, según la tabla 4, confirma la efectividad del programa en la mejora del nivel de prácticas sobre alimentación saludable y actividad física con un valor de sig $=0.001$, comparado con el valor del nivel de significancia (0.05); por lo tanto, el programa fue efectivo en mejorar el nivel de prácticas en los estudiantes que participaron en el programa.

\section{DISCUSIÓN}

El diagnóstico del Índice de Masa Corporal, sobrepeso y obesidad, prevaleció casi en la mitad del total de los escolares (41.1\%) el cual es un mensaje de alarma para la institución educativa, debido a que el desarrollo del escolar no será óptimo.

Por otro lado, se encontró un caso de riesgo de un esco- lar con obesidad y talla baja, debido posiblemente a la mala distribución de alimentos energéticos y proteicos. Casos como éste deben ser atendidos lo antes posible, para que atenuar los daños irreversibles.

Al comienzo de la intervención educativa los resultados de la encuesta realizada en escolares solo alcanzaron el $52.9 \%$ de buenas prácticas de hábitos alimentarios y actividad física; sin embargo, al finalizar la interven- 
ción el $100 \%$ de los escolares tuvo buenas prácticas, esto se pudo comprobar en las loncheras que traían o en las compras que realizaban en el kiosco. Asimismo, se brindó información sobre lonchera saludable a la propietaria del kiosco, a fin de promover la venta de productos saludables o la modificación de algunas de los alimentos preparados como por ejemplo; pan con queso o tortilla de verduras, galletas integrales, refresco de fruta con moderado azúcar.

En la dimensión de conocimientos, los escolares refirieron no tener buena base sobre una buena alimentación, lo que reflejó el resultado del pre test, donde el $100 \%$ tenían malos conocimientos, lo que más adelante después de la intervención mejoró significativamente a $82.4 \%$ con buenos conocimientos. A pesar de la edad, los escolares demuestran conocer los efectos negativos en la salud del consumo elevado de alimentos azucarados, golosinas o preparaciones rápidas.

Arrivillaga (2003), refiere que aunque el conocimiento, por sí solo, no es indicador de cambio conductual, sí se ha demostrado que cierta cantidad de información es necesaria para realizar el proceso que conducirá a un cambio de comportamiento.

De forma similar, Long (1999), menciona que es necesario brindar información oportuna y completa sobre alimentación saludable y actividad física porque los protegerá de enfermedades más adelante.

En la dimensión de actitud, los cambios fueron importantes, antes de la intervención el $23.5 \%$ presentaban actitudes negativas hacia la alimentación saludable y actividad física. Después de la intervención el 100\% presentaron actitudes positivas. Cabe resaltar que algunos alumnos presentaban rechazo hacia algunas frutas y verduras antes de la intervención, pero tenían mayor gusto por las golosinas y jugos comprados o gaseosas. Pero al término del programa cambiaron de parecer eligiendo alimentos saludables.

La actitud es el vínculo del conocimiento adquirido por una persona y la acción de la misma en el presente y el futuro; una actitud es una disipación mental y neurológica que se organiza a partir de la experiencia y que ejerce una directriz o una dinámica sobre las reacciones del individuo, respecto de todos los objetos y a todas las situaciones que le corresponde.

En cuanto a las prácticas el $52.9 \%$ de los escolares presentaban malas prácticas de alimentación saludable y actividad física antes de la intervención. Después del programa, el $100 \%$ tuvieron prácticas buenas.

La práctica es la actividad de refuerzo que da al aprendiz la oportunidad de aplicar conocimientos y habilidades. Este sistema suele incluir tutorías y retroalimentación (Poletti, Oscar \& Barrios, 2003).

\section{CONCLUSIONES Y RECOMENDACIONES}

- El programa educativo "Healthy Kids" fue efectivo en los conocimientos, actitudes y prácticas sobre la alimentación saludable y actividad física en los alumnos del $3^{\circ}$ grado de primaria y sus madres del Colegio Almirante Guillermo Brown, en el distrito de Villa El Salvador.

- Se recomienda que la evaluación nutricional a los niños debe ser trimestralmente o semestralmente, solicitando ayuda de un ente de salud. Por otro lado, se debe insertar este programa al comienzo de cada año escolar. 


\section{REFERENCIAS BIBLIOGRÁFICAS}

1. Adolfo, O, Jáuregui M. Ruiz D. (2007). Efectos de programa de intervención en sobrepeso y obesidad de niños escolares en Colima, México. Salud Public Méx. Nov-Dic 49:6 pp 389-392.

2. Arrivillaga, M., Salazar, I., \& Correa, D. (agosto, 2003). Creencias sobre la salud y su relación con las prácticas de riesgo o de protección en jóvenes universitarios. Revista Colombia Médica, 40-50. Recuperado de http://redalyc.uaemex.mx/src/inicio/ ArtPdfRed.jsp?iCve=28334403.

3. Instituto de Nutrición y Tecnología de los Alimentos. (2005) Obesidad desde la infancia. INTA.

4. Llanos F, Cabello E. (octubre, 2003). Distribución del índice de masa corporal (IMC) y prevalencia de obesidad primaria en niños pre-púberes de 6 a10 años de edad del distrito de San Martín de Porres - Lima. Revista Médica Herediana Peruana 14 (3): 107- 110.

5. Lloyd, L. (2003). Comunicación y cambio conductual. Recuperado de http://www.ipk.sld.cu/ curso.

6. Organización Mundial de la Salud. (2005). Obesidad Recuperado de: http://www.who.int/mediacentre/ news/releases/2005/pr44/es/index.html.

7. Organización Mundial de la Salud. (2006). Estrategia Mundial sobre Régimen Alimentario,
Actividad Física y Salud. Recuperado de: http:// www.who.int/dietphysicalactivity/childhood/en/ index.html.

8. Organización Panamericana de la Salud (2003). Régimen alimentario, nutrición y actividad física. Washington DC: OPS.

9. Pérez L., Raigada J., M. Collins A., Mauricio A. Antonio Felices P. Sofía Jiménez C. et al. (2008). Efectividad de un programa educativo en estilos de vida saludables sobre la reducción de sobrepeso y obesidad en el Colegio Robert M. Smith; Huaraz, Ancash, Perú. Act Med Per (online). Oct-Dic 2008 (citado 18 de octubre) 25(4): 204-209 pp. Recuperado de: http://www.scielo.org.pe/scielo. php?pid $=$ S1728-9172008010000004\&script $=$ sci arttext.

10. Poletti C., Oscar H. \& Barrios M. L. (septiembre, 2003). Sobrepeso y obesidad como componentes de la malnutrición, en escolares de la ciudad de Corrientes. Argentina. Revista. Chile. pediatr. v.74 n.5 Santiago.

11. Styme D.M: Childhood and adolescent obesity. Prevalence and significance. Pediatr Clin North Am 2001; 48: 823-54.

12. Summerbell CD, Waters E, Edmunds LD, Kelly S, Brown T, Campbell KJ. (2008). Intervenciones para prevenir la obesidad infantil. Biblioteca Cochrane plus. 1:2 pp 1-74. 